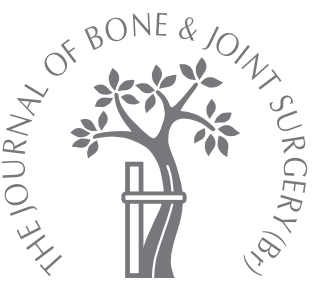

K.-Y. Huang,

R.-S. Yang,

C.-C. Hsieh

From National

Cheng Kung

University Hospital,

Tainan, Taiwan
K.-Y. Huang, MD, Orthopaedic Surgeon, Assistant Professor

Department of Orthopaedics and Institute of Clinical

Medicine, College of Medicine C.-C. Hsieh, MD, Diagnostic Radiologist

Department of Radiology

National Cheng Kung

University Hospital, Tainan,

Taiwan No. 138 Sheng Li Road,

Tainan City 70428, Taiwan.

R.-S. Yang, MD, PhD

Orthopaedic Surgeon,

Professor

Department of Orthopaedics,

College of Medicine

National Taiwan University

Medical Center, No. 7 Chung

Shan South Road, Taipei 10043 ,

Taiwan.

Correspondence should be sent to Dr C.-C. Hsieh; e-mail: goba.goba@msa.hinet.net

(C)2009 British Editorial Society of Bone and Joint Surgery doi:10.1302/0301-620X.91B9. $22276 \$ 2.00$

$J$ Bone Joint Surg $[\mathrm{Br}]$ 2009;91-B:1249-51. Received 7 January 2009; Accepted after revision 7 April 2009

\title{
Simultaneous osteonecrosis and osteomyelitis in a patient with cancer of the breast
}

\begin{abstract}
Breast cancer is generally managed surgically with adjuvant agents which include hormone therapy, chemotherapy, radiotherapy and bisphosphonate therapy. However, some of these adjuvant therapies may cause adverse events, including wound infection, neutropenia, bone marrow suppression and fever. The simultaneous presentation of osteonecrosis and osteomyelitis has not previously been described in patients with breast cancer undergoing hormone therapy and chemotherapy.

We report a patient with breast cancer who developed bone infarcts in both legs as well as osteomyelitis in the right distal tibia after treatment which included a modified radical mastectomy, hormone therapy and chemotherapy. Simultaneous osteonecrosis and osteomyelitis should be considered in patients with breast cancer who are receiving chemotherapy and hormone therapy who present with severe bone pain, especially if there have been infective episodes during treatment.
\end{abstract}

Carcinoma of the breast is a common malignancy. In addition to surgery, non-surgical treatments such as hormone therapy, chemotherapy, radiation therapy and use of bisphosphonates improve the prognosis, ${ }^{1}$ but may be associated with adverse events, including problems with wound healing, neutropenia, anaemia and fever. ${ }^{2,3}$ Careful follow-up is required. ${ }^{4,5}$ Simultaneous presentation of bone infarcts and osteomyelitis has not been reported in patients with breast cancer undergoing hormone therapy and chemotherapy. We report such a case and discuss the difficulties in making the diagnosis.

\section{Case report}

In April 2006 a 37-year-old woman presented with an invasive ductal carcinoma of the left breast, stage T2N3M0, and underwent a modified radical mastectomy. This was followed by five courses of chemotherapy with a regimen of taxotere, epirubicin and exdoxan, as well as hormone therapy comprising zoladex and tamoxifen because of the presence of positive oestrogen and progesterone receptors. She developed a wound infection about two months after operation, accompanied by two episodes of neutropenic fever during chemotherapy. In September 2006 split skin grafting was performed for wound dehiscence. An episode of cellulitis of the right shin accompanied by methicillin-resistant Staphylococcus aureus
(MRSA) bacteraemia and focal abscess formation (also on the shin) occurred in March 2007. The condition healed after incision, debridement and antibiotic therapy with cefazolin and gentamicin for two weeks. However, in April 2007, there was a recurrence of a hot painful swelling on the right shin. The white blood cell count was $3900 / \mathrm{ml}$, of which $68.3 \%$ were neutrophils. The CRP was elevated to $13.9 \mathrm{mg} / \mathrm{L}$. She developed diabetes in May 2007 with an elevated post-prandial blood sugar of $245 \mathrm{mg} / \mathrm{dl}$ and fasting blood sugar of $126 \mathrm{mg} / \mathrm{dl}$, and oral treatment was started. She was assessed for evidence of immunocompromise by rapid plasma reagin, a VDRL test and an anti-HIV antibody test, all of which were negative.

Further investigation, including abdominal and breast ultrasound, excluded liver metastases or recurrent breast cancer.

A technetium- $\left({ }^{99 \mathrm{~m}} \mathrm{Tc}\right)$ methylene diphosphonate $\left(\mathrm{T}^{-99} \mathrm{~m} \mathrm{Tc}-\mathrm{MDP}\right)$ radionuclide bone scan revealed increased uptake of isotope in the right tibia suggestive of osteomyelitis. Cefazolin and gentamicin had been prescribed empirically since May 2007 after admission. A plain radiograph of the right tibia revealed an ill-defined osteolytic lesion in the distal medullary canal with a surrounding continuous periosteal reaction (Fig. 1). These appearances suggested subacute or chronic osteomyelitis. A retrospective review of the previous plain radiographs 

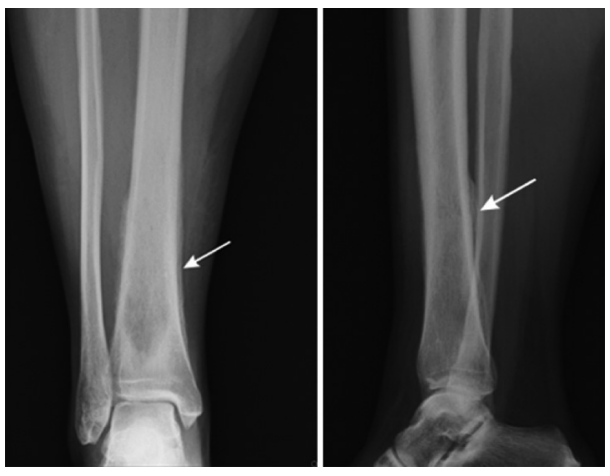

Fig. 1

Anteroposterior and lateral radiographs of the right tibia showing an ill-defined osteolytic lesion with continuous periosteal reaction (arrows) distally. Osteomyelitis or bony metastasis should be considered in the differential diagnosis according to the radiological findings.

from April 2007 also revealed an ambiguous osteolytic lesion at same site, accompanied by a mild periosteal reaction (Fig. 2). An MRI revealed oedematous change in the right distal tibial bone marrow with enhancement extending to the periosteal region (Fig. 3). In addition, an incidental finding of serpinginous osteonecrosis in both distal femora and proximal tibiae was observed (Fig. 4). T- ${ }^{99 \mathrm{~m}} \mathrm{Tc}-\mathrm{MDP}$ three-phase bone scanning showed increased blood flow and blood pooling in the right tibia. Delayed images showed an increased radioactivity in the right tibia. A follow-up gallium-67 $\left({ }^{67} \mathrm{Ga}\right)$ scan showed increased radioactivity in the right tibia. These results suggested osteomyelitis of the right tibia. Consequently, debridement and sequestrectomy of the right distal tibia were performed, during which subcutaneous thickening, destruction of the bone with cortical thinning and erosion, friable tissue in the intramedullary cavity and a fibrous membrane were found. A synthetic bone substitute mixed with vancomycin powder was inserted into the bony defect. Post-operatively a long leg brace was applied for six weeks. Bacterial culture yielded MRSA confirming the diagnosis of chronic osteomyelitis.

She was discharged on rifampin $300 \mathrm{mg}$ twice daily and $1 \mathrm{~g}$ fucidin three times daily, plus alendronate $70 \mathrm{mg}$ per week (to prevent bony destruction) for four months. The incision healed satisfactorily after two weeks and the ESR and CRP returned to normal within three months. Followup radiographs of the right leg five months post-operatively revealed regression of the periosteal reaction, and a followup radiograph at one year demonstrated stable healing of the previous lesion without any periosteal reaction. An MR scan at this stage revealed intramedullary sclerosis at the site of the previous osteomyelitis with peripheral reactive change. Neither a discernible periosteal reaction nor softtissue enhancement around the right tibia was present. Follow-up T- ${ }^{99 \mathrm{~m}} \mathrm{Tc}-\mathrm{MDP}$ and ${ }^{67} \mathrm{GA}$ scanning identified minimal activity with markedly reduced intensity com-
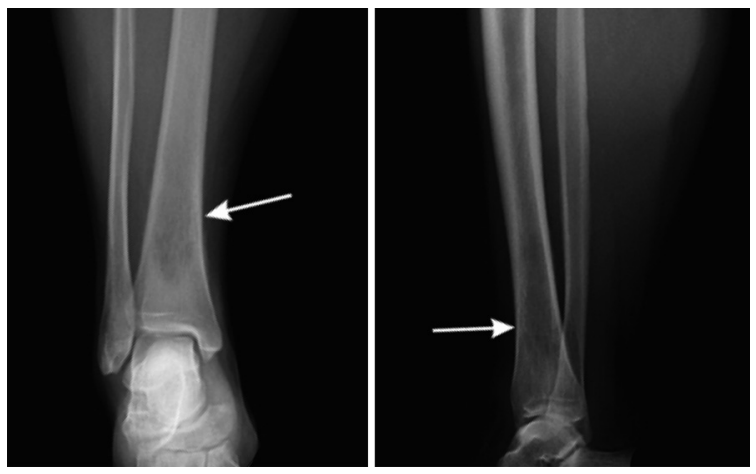

Fig. 2

Anteroposterior and lateral radiographs of the right tibia obtained during the previous admission for cellulitis in April 2007 showing an ill-defined osteolytic lesion with mild continuous periosteal reaction distally around the medial aspect. This was the site of the subsequent osteomyelitis.

pared with the pre-operative studies, reflecting residual reactive and reparative change.

\section{Discussion}

Carcinoma of the breast is associated with a relatively high risk of metastasis to bone. ${ }^{2-5}$

Non-traumatic osteonecrosis is a well-documented late complication of chemotherapy for haematological malignancies; prolonged corticosteroid exposure has been suggested as a possible underlying cause. ${ }^{1}$ A systematic review of non-traumatic osteonecrosis after chemotherapy for solid tumours found that most reported cases occurred in adults with testicular or breast cancer. ${ }^{2,6} \mathrm{~A}$ further report detailed the development of osteonecrosis of the femoral head in breast cancer after adjuvant chemotherapy. ${ }^{7}$ There is a single report of four cases with osteomyelitis originating in and around bone infarcts in the femora. ${ }^{8}$ Other reports describe osteomyelitis of the lunate in a patient with bilateral Kienböck's disease, ${ }^{9}$ one case of Candida albicans osteomyelitis in a patient with avascular necrosis of the hip, ${ }^{10}$ and one case of mucormycosis osteomyelitis causing osteonecrosis of the cuboid bone ${ }^{11}$ has been reported. However, patients with breast cancer undergoing surgical treatment, hormone therapy and chemotherapy have not, to date, been reported to develop simultaneous multifocal osteonecrosis of bilateral long bones and osteomyelitis of the tibia.

It is accepted that early diagnosis of osteomyelitis is essential to avoid abscess formation, deformity and pathological fracture, but the radiological features are difficult to identify in its early stages. Plain radiographs do not usually show any abnormality until 10 to 14 days after the onset of osteomyelitis. ${ }^{12,13}$ In contrast, a radionuclide bone scan may exhibit abnormal findings as early as three days after onset.

Although osteonecrosis after chemotherapy occurs rarely in patients with breast cancer, ${ }^{7}$ bone infarcts of both distal 


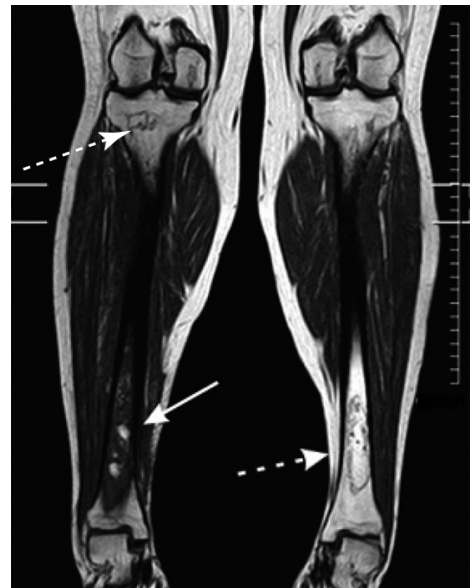

Fig. 3a

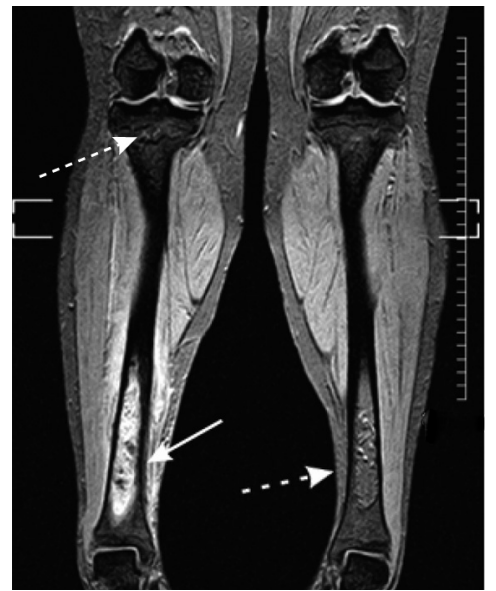

Fig. 3b

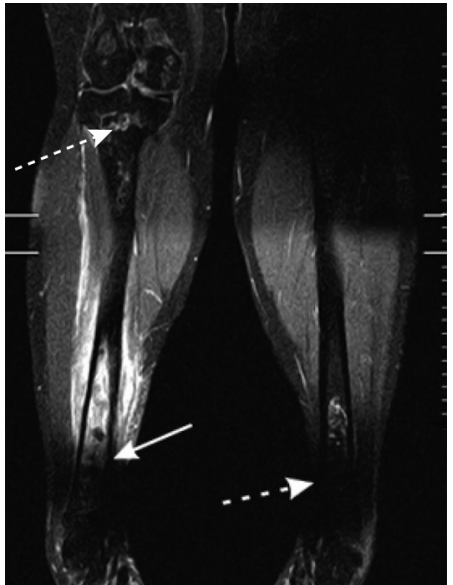

Fig. 3c

Coronal MR scans of both legs a) T1-weighted, b) T2-weighted with fat suppression, and c) T1-weighted with fat suppression after intravenous gadolinium injection. There is a focal lesion in the bone marrow cavity of the distal right tibia, with hypointense signal on T1 and hyperintense signal on T2 with enhancement, and also enhancement around the periosteal region of the right tibia compatible with osteomyelitis (white arrows). Additionally, the bone infarcts were shown in the right proximal tibia and left tibia (dotted white arrows).
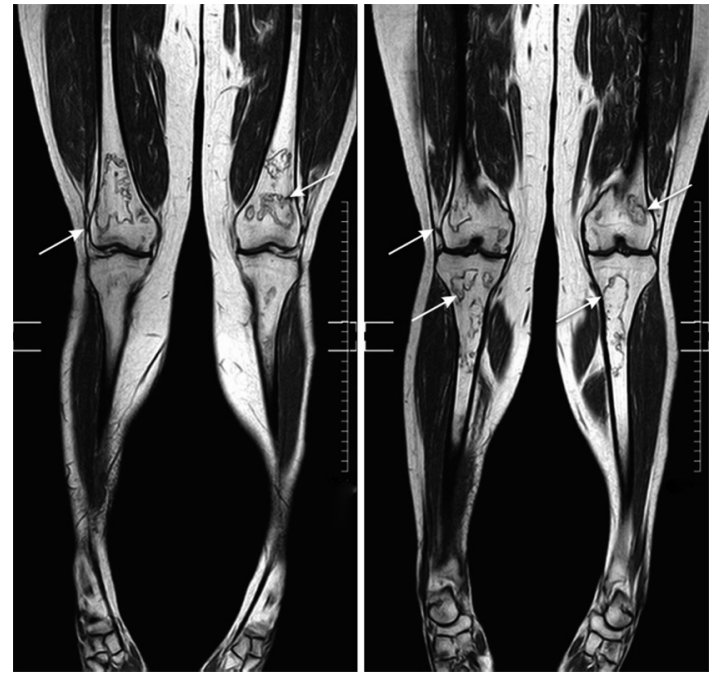

Fig. 4

Coronal T1-weighted MR scans of both legs. There were irregular well-defined areas with low-signal margins in the bilateral distal femora and proximal tibiae (arrows).

femora and proximal tibiae were noted incidentally on MRI during our investigations for osteomyelitis. The MRI features of a bone infarct are a well-defined area with similar signal intensity to the normal marrow, with a low-signal band on T1-weighted images and a hyperintense rim at the periphery producing a double-line sign on T2-weighted images. ${ }^{13}$ The typical serpiginous shape and signal intensity may help to differentiate it from a bony metastasis. In this unusual case, the bone infarcts may have been related to the hormone therapy or chemotherapy, but the coexistence of right tibial osteomyelitis was probably the result of bacteraemia during a period of immune compromise whilst undergoing chemotherapy when she developed a wound infection and two episodes of neutropenic fever.

In conclusion, osteonecrosis in combination with osteomyelitis is rare but should not be overlooked when treating patients with breast cancer for presumed metastases.

No benefits in any form have been received or will be received from a commercial party related directly or indirectly to the subject of this article.

\section{References}

1. Hirbe A, Morgan EA, Uluckan 0, Weibaecher K. Skeletal complications of breast cancer therapies. Clin Cancer Res 2006;12(Suppl 20):6309-14.

2. Colleoni M, O'Neill A, Goldhirsch A, et al. Identifying breast cancer patients at high risk for bone metastases. J Clin Oncol 2000;18:3925-35.

3. Front D, Schneck SO, Frankel A, Robinson E. Bone metastases and bone pain in breast cancer: are they closely associated? JAMA 1979;242:1747-8.

4. Palmer E, Henrikson B, McKusick K, Strauss HW, Hochberg F. Pain as an indicator of bone metastasis. Acta Radiol 1988;29:445-9.

5. Hortobagyi GN. Treatment of breast cancer. N Engl J Med 1988;339:974-84

6. Winquist EW, Bauman GS, Balogh J. Nontraumatic osteonecrosis after chemotherapy for testicular cancer: a systematic review. Clin Oncol 2001;24:603-6.

7. Dawson LK, Nussey F, Oliver TB, Marks RC, Leonard RC. Osteonecrosis of the femoral head following adjuvant chemotherapy for breast cancer. Breast 2001;10:447-9.

8. Blacksin MF, Finzel KC, Benevenia J. Osteomyelitis originating in and around bone infarcts: giant sequestrum phenomena. AJR Am J Roentgeno/ 2001;176:387-91.

9. Kahn ML, Bade HA. Lunate osteomyelitis in a patient with bilateral Kienböck's disease. Orthop Rev 1986;15:521-5.

10. Cooper P, Schofield B, Lennox DW, Ebert-Smith T. Candida albicans osteomyelitis in a patient with avascular necrosis of the hip. Orthopedics 1991;14:352-5.

11. Chaudhuri R, McKeown B, Harrington D, et al. Mucormycosis osteomyelitis causing avascular necrosis of the cuboid bone: MR imaging findings. AJR Am J Roentgenol 1992;159:1035-7

12. Ma LD, Frassica FJ, Bluemke DA, Fishman EK. CT and MRI evaluation of musculoskeletal infection. Crit Rev Diagn Imaging 1997;36:535-8.

13. Munk PL, Helms CA, Holt RG. Immature bone infarcts: findings of plain radiographs and MR scans. AJR Am J Roentgenol 1989;152:547-9. 